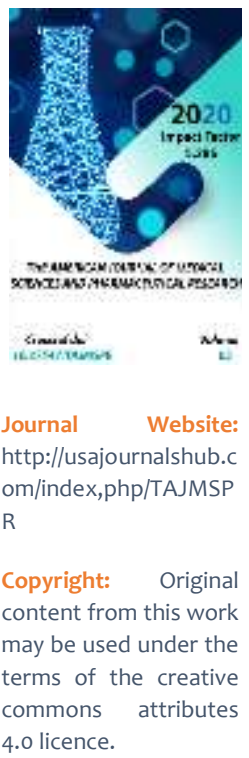

\title{
Congestion Of Lung Assessment Examination Of Endoscopic Technique And Nonendoscopic Technique Procedures
}

\author{
Ananta Khurana \\ Professor, Departments Of Medicine, Gandhi Medical College, Bhopal, India \\ Kabir Sardana, \\ Departments Of Medicine, Gandhi Medical College, Bhopal, India
}

\section{ABSTRACT}

The conclusion of fan related congestion of lung stays a test in light of the fact that the clinical signs and side effects need both affectability and explicitness and the choice of microbiologic demonstrative technique is as yet a matter of discussion. Points and Target: To contemplate the job of different endoscopic technique and nonendoscopic technique indicative methods for analysis of FRC. Settings and Structure: This imminent relative examination was directed in a clinical ICU of a tertiary consideration place. There was a decent microbiologic concordance among various endoscopic technique and non-endoscopic technique distal aviation route inspecting strategies. End: NBAL is a modest, simple, and valuable procedure for microbiologic conclusion of FRC. Our discoveries, whenever confirmed, might improve the methodology for the conclusion of FRC.

\section{KEYWORDS}

Endoscopic technique brush, Broncho alveolar lavage(BAL), endotracheal suction, nonendoscopic technique broncho alveolar lavage, fan related congestion of lung.

\section{INTRODUCTION}

The conclusion of fan related congestion of lung stays a test on the grounds that the clinical signs and manifestations need both affectability and particularity and the choice of microbiologic symptomatic system is as yet only debate.[1] Clinically, FRC is characterized by four measures: (1) the radiographic appearance of another or dynamic aspiratory 
penetrates, (2) fever, (3) leukocytosis, and (4) purulent tracheobronchial secretions.

Exact clinical and microbiologic determination of FRC is fundamental for choice of proper antimicrobials as well as to forestall their abuse. Improper utilization of antimicrobials in these patients prompts expanded mortality and development of multidrug safe microorganisms in the ICU. It has been proposed by various examiners that "intrusive" symptomatic strategies, including quantitative societies of distal aviation route examples got by utilizing endoscopic technique broncho alveolar lavage (BAL), endoscopic technique brush, secured BAL, or ensured example brush (PSB), could improve distinguishing proof of patients with genuine FRC and choice of fitting antibiotics.In any case, bronchoscopy requires specialized aptitude and adds to the expense of care. The consequences of the examinations utilizing endoscopic technique strategies are conflicting, demonstrating both bogus positive and bogus negative outcomes, which further inquiries their accurate job in the analysis of FRC. Trying to beat these restrictions, nonendoscopic technique distal aviation route examining strategies have risen, as nonendoscopic technique BAL (NBAL) and nonendoscopic technique PSB. In spite of the fact that straightforward and cheap, symptomatic precision of these visually impaired examining strategies has not been concentrated in the Indian setting.

\section{MATERIALS AND TECHNIQUES}

This imminent similar investigation was led in the clinical emergency unit of a tertiary consideration place arranged in northern India. The examination was affirmed by the institutional survey board. In the wake of acquiring composed educated assent from either the patient or the first degree relative, we enlisted 25 patients old enough 18 years or more who required fany help for the former 48 hours or more with clinical and radiological conclusion of FRC. Additionally, patients who required mechanical fan in another emergency clinic for 48 hours or more before admission to our establishment and the individuals who were extubated/weaned or passed on inside 3 days of intubation were likewise avoided from the examination. For every patient contemplated, these boundaries were recorded: age, sexual orientation, essential determination on affirmation, sign of mechanical ventilation, comorbid conditions.

\section{Endotracheal Suction And Non-Endoscopic Technique BAL}

The Estimated time of arrival examples were gathered by means of a sputum pull trap. Nonendoscopic technique ensured BAL was performed by twofold catheter method. A sterile pull catheter of size $16 \mathrm{Fr}$ was cut $2-3 \mathrm{~cm}$ from the distal end to give a last length of around $47-48 \mathrm{~cm}$ and embedded through the endotracheal tube and aimlessly progressed into the distal aviation routes till opposition is felt. The catheter was wedged in that position, and a second, 50-cm long, sterile attractions catheter of size $8 \mathrm{Fr}$ was gone through the primary catheter and progressed beyond what many would consider possible. Twenty milliliters of ordinary saline was imparted into the distal aviation routes through the internal cylinder and suction was gathered in a sterile holder. Amount of the suction was recorded. System was rehashed if the suctioned liquid was under $5 \mathrm{ml}$. The examples were quickly moved for bacteriologic assessment and quantitative societies. 


\section{Endoscopic Technique BAL And Brush}

Patients were calmed with $5 \mathrm{mg}$ of intravenous midazolam. The fany settings were balanced by expanding flowing volume by $100 \mathrm{ml}$ and $\mathrm{FiO}_{2}$ to 1.o. All the vitals including pulse, circulatory strain and oxygen immersion were observed utilizing persistent heartbeat oxymetry during the whole methodology. also, the tip was situated near the hole of the bronchus, depleting the broncho pulmonary section of enthusiasm as dictated by chest radiograph. In patients with diffuse/two-sided lung invades, bronchoscope was progressed into a broncho pulmonary section of the correct lower projection for testing. On arriving at the territory to be examined, the brush was jutted, dove various occasions into the presumed bronchus, and pulled back. The example was then moved by mixing the brush into a sterile vial containing $1 \mathrm{ml}$ of ordinary saline which was utilized for microbiologic examination.

The affectability, explicitness, positive prescient worth (PPV) and negative prescient worth (NPV) of nonendoscopic technique secured $B A L$, endoscopic technique $B A L$ and endoscopic technique brushings, for the conclusion of FRC, were determined taking CPIS score of 6 as reference standard.

\section{RESULTS}

Ceaseless obstructive aspiratory illness with type-II respiratory disappointment was the commonest sign of mechanical ventilation. Different infections incorporate metabolic encephalopathy (2), cerebral vascular sickness (2), intense provocative demyelinating polyneuropathy (1), intense spread encephalomyelitis (1), interminable liver illness (1), myotonic solid dystrophy (1), organo phosphorus harming (1), constant renal disappointment with frailty (1), and sub-dural hematoma because of street car crash (1). Dominant part of patients, aside from two cases, were analyzed to have late beginning FRC.

\section{CONVERSATION}

FRC is a typical confusion related with intrusive fan backing and adds to a significantmortality and bleakness in these patients. In view of helpless particularity of the clinical conclusion of FRC, dependence is frequently positioned on radiologic and microbiologic finding. Microbiologic conclusion involves culture of blood, pleural liquid and respiratory emissions including proximal (tracheal suction) and distal aviation routes (BAL and brush). It is imperative to remember that the affectability of blood culture for determination of FRC is under $25 \%$, and in any event, when positive, the living being may start from an extrapulmonary site of disease in the same number of as $64 \%$ of cases, in any event, when FRC is available.

Estimated time of arrival is the most generally utilized strategy for aviation route testing in ICUs everywhere throughout the world. Gram stain, non quantitative and semi-quantitative culture of tracheal emissions has the upside of reproducibility and of requiring minimal specialized aptitude and no specific hardware or strategy. In any case, these investigations add little to the affectability and explicitness of the clinical finding of FRC, as the upper respiratory tract is every now and again colonized with likely microbes, even without congestion of lung. the ideal methodology for the finding of FRC stays to be characterized. The American Thoracic Culture rules do give master conclusion supporting quantitative or semi-quantitative societies of respiratory 
examples, in spite of the fact that the board favors intrusive quantitative strategies.

\section{CONCLUSION}

Our investigation has demonstrated that NBAL is a worthy option to bronchoscopy for the assessment of suspected FRC. In this way, our perceptions, whenever checked in different ICUs, may streamline the methodology for the analysis of FRC. This end depends on the way that NBAL is moderately cheap, requires lesser aptitude, and might be a valuable strategy for the sequential assessment of suspected nosocomial congestion of lung in patients on mechanical ventilation.

\section{REFERENCES}

1. Correia JC, Golay A, Lachat S, Singh SB, et al. Explanation of the fourth Worldwide Accord Meeting in Basic Consideration on ICU-Procured Congestion of lung- Chicago, Illinois, June 2008. Serious Consideration Medications 2008;32:22136.

2. Amatya R, Adhikari S, Giri D,. Nosocomial respiratory contaminations with gramnegative bacilli. The centrality of colonization of the respiratory tract. Ann Understudy Drug 1982;24:321-336.

3. Narang T, Kaur I, Kumar B, Radotra BD, Analysis of nosocomial bacterial congestion of lung in intense, diffuse lung injury. Chest 1985;34:744-758.
4. Job CK, Yoder L, Jacobson RR, Hastings RC. Assessment of clinical judgment in the recognizable proof and treatment of nosocomial congestion of lung in ventilated patients. Chest 1995;210:647653.

5. Murashov MD, Diaz-Espinosa J, Finding of fan-related congestion of lung by bacteriologic investigation of endoscopic technique and nonendoscopic technique"dazzle" broncho alveolar lavage liquid. Am Fire up Respir Dis 1994;213:3101-3129.

6. Arora P, Sardana K, Agarwal A, Lavania $M$. Short-course empiric anti-toxin treatment for patients with aspiratory invades in the emergency unit. A proposed answer for unpredictable antiinfection solution. Am J Respir Crit Care Drug 2007;212:105-101. 